\title{
An Internet of Intelligences with WebGIS Constructed by Leaflet Design
}

\author{
Yannan Sun ${ }^{1,2,3}$, Chongfu Huang ${ }^{1,2,3, *}$ \\ ${ }^{1}$ Key Laboratory of Environmental Change and Natural Disaster, Ministry of Education, Beijing \\ Normal University, Beijing 100875, China \\ ${ }^{2}$ State Key Laboratory of Earth Surface Processes and Resource Ecology, Beijing Normal \\ University, Beijing 100875, China \\ ${ }^{3}$ Faculty of Geographical Science, Academy of Disaster Reduction and Emergency Management, \\ Beijing Normal University, Beijing 100875, China
}

\section{由 Leaflet 构建网络 GIS 支持的智联网 孙雁南 ${ }^{1,2,3}$, 黄崇福 ${ }^{1,2,3, *}$ \\ ${ }^{1}$ 北京师范大学，环境演变与自然灾害教育部重点实验室，北京 100875 , 中国 ${ }^{2}$ 北京师范大学, 地表过程与资源生态国家重点实验室, 北京 100875 , 中国 \\ ${ }^{3}$ 北京师范大学, 地理科学学部, 减灾与应急管理研究院, 北京 100875 , 中国}

\begin{abstract}
Based on the characteristics of Leaflet, this paper builds a WebGIS to support an Internet of intelligences (IOI), which does not depend on any third-party plug-ins, enhances the ease of deployment and adaptability to different platforms. Such WebGIS enhances the convenience of deployment and adaptability to different platforms. Then, an IOI more easy to use geographic information and quickly collect, process, and analyze disaster risk data.This article demonstrates how such an IOI can be used for vulnerability assessment.
\end{abstract}

Keyword: Internet of intelligences, WebGIS, Leaflet, Vulnerability

\section{摘要}

本文基于 Leaflet 的特性, 搭建了不依赖任 何第三方插件的, 支持智联网的网络 GIS。此

基金项目: 国家自然科学基金项目 (编号: 41671502), 国家重点研发计划（编号：2017YFC1502902）。 *通讯作者: hchongfu@bnu. edu. cn
网络 GIS 增强了部署的便捷性和对不同平台 的适应性。从而, 智联网可更方便地使用地 理信息, 并快速收集、处理与分析灾害风险 数据。本文演示了这样的智联网如何用于脆 弱性评价。

关键词: 智联网; 地理信息服务; Leaflet; 脆弱性

\section{1. 地理信息服务和智联网发展现状}

\section{1 网络地理信息服务}

随着地理信息系统(GIS)学科的发展, 地 理信息系统正在向地理信息服务偏转 [1]。地 理信息服务概念的初衷是为了提高地理信 息系统和资源的利用率。地理信息服务就是 对地理信息提供的服务, 包括地理数据服务 和地理信息系统信息处理功能的服务[2]。国 内网络地理信息服务的开发和应用方面处 在不断发展的阶段, 主要集中在行业部门级 的应用、政府主导的公众基础网络地理信息 服务共享平台的建设 [3], 例如旅游、购物、 交通信息等。《2001 年度中国软件产业发展 
研究报告》(中国软件行业协会) 中也明确 指出: “GIS 正在从专业技术领域走向社会 化地理信息服务”。徐永龙(2011)采用 Flex 作为客户端采用 HTTP Server 和 Web (网 络)Server 的方式与服务器通信实现了 WebGIS[4]。不少城市开始尝试利用以网络 化地图与地理信息服务等方式扩宽参与公 众决策的途径, 使得公众利益互联网的便捷 来获取有效信息和发表建议, 如, 西安市高 新区地理信息系统公众服务平台, 为市民提 供专题地图、便民服务等。

目前, 国内外大多数构建和调用地理信 息服务的商业软件价格昂贵, 开发成本高[5]。 由于地理信息系统开发涉及的学科领域多、 周期长、内容复杂, 商业 GIS 平台价格高昂, 特别针对于是中小型用户对地理信息系统 的需求往往只是需要其中的一部分功能, 并 不需要花费巨大的成本来购买整个商业平 台, 而开源 GIS 的出现则满足了在不购买商 业平台基础上的开发功能的技术需求 [6]。

众多的 GIS 爱好者致力于开源 GIS 软件的研发, 从而使得开源 GIS 软件问世。 目前, 开源 GIS 软件已经形成了一个比较 齐全的产品线, 当前存在各种层次的开源的 GIS 软件, 例如大型的桌面软件有 QGIS、 GRASS GIS 等, 比较流行的服务器端软件有 Geoserver、MapServer 和 QGIS Server 等, 开源数据库项目 PostGIS/PostgreSQL Spatial Database, 数据转换工具 OGR 和 GDAL 等 以及地图投影算法库 Proj4 和 Geotrans 等开 源项目 [7]。基于开源 GIS 软件的开发应用 项目越来越广。

\section{2 智联网}

智联网的最初思想是 2011 年黄崇福在 研究如何进行风险分析在线服务时产生的 [8]。通俗来说, 智联网是由互联网联结具有 观察、演绎、推理和解决问题能力的多个人 (智能体), 并通过嵌入的模型集个体小智 慧为群体大智慧的网络。假设 $A$ 是一个智能 体集合, $N$ 是 $A$ 使用的一个网络, $M$ 是处 理 $A$ 所提供信息的模型, 三元体 $\langle A, N, M>$ 称为一个智联网 [9]。

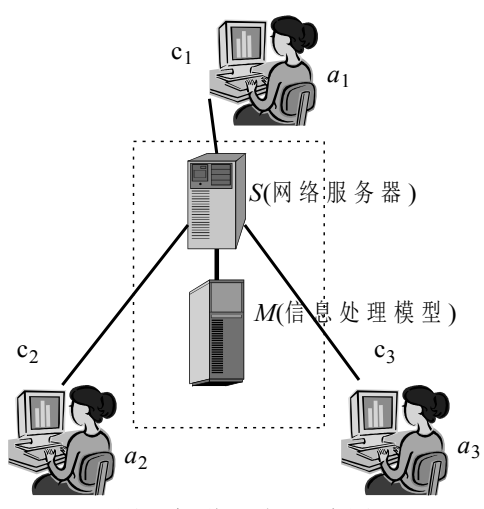

图 1 智联网原理示意图

在自然灾害风险分析领域已有各种关 于灾害的研究方法与体系, 但由于承灾体破 坏机理的复杂性及多样性, 很多都处于假说 阶段或者尚未完全清楚, 大大降低了样本数 据的获取数量与精度, 并且在输入与输出关 系上, 只能表达为多值对应的关系, 而非一 对一的输入-输出关系, 因此在此基础上进行 的脆弱性分析是不确定的。

智联网的智能活动具有传统的灾害风 险数理分析无法替代的优点, 黄崇福教授 2012 年特地讨论了, 利用智联网的柔性感知 原理可避开统计假设、网络互连为获取足够 多的数据信息、实时更新捕捉风险动态提供 了可能, 从而可有效解决传统风险分析假设 规律、数据获取难、风险时时变化、不确定 性等四大难题[10]。一方面, 充分利用网络 的便利性, 为信息经验丰富的智能体提供了 智慧相互传递、交流的平台, 从而集小智慧 为大智慧, 创造了风险分析的网络化方式; 另一方面, 以信息扩散算法 [11]为基础的数 学模型, 利用模糊关系来表达样本数据的不 确定关系。

\section{2. 开发工具的选择}

本文选取开源 GIS 工具 QGIS 和 Leaflet, 阐述了它们的特点以及关键技术, 提出构建和调用地理信息服务的体系框架。

\subsection{Leaflet}

Leaflet 是一个开源的 WebGIS Java Script 库, 其源代码仅有 $33 \mathrm{~KB}$, 但它具有 开发在线 WebGIS 的大部分功能[12]。具有 良好的交互性与移动端设备的适用性, 不管 是在主要桌面还是移动设备山都可以高效 运行。Leaflet 支持插件的扩展, 其开源插件 库涉及到地图服务、数据支持、地理编码、 
地图空间、路线、交互等各个方面, 同时也 支持自定义控件对 Leaflet 实现功能的扩展。

Leaflet 的框架结构主要分为以下八类, 采用与 jQuery 链式编码类似的风格, 具有代 码简单易读的优势。Leaflet 源码文件的组织 结构表见表 1 。
支撑的功能扩展, (5)支持与数据库的连接加 载, 支撑各类数据格式的显示和加载。特别 是在对多种数据格式的支持上, QGIS 具有 显著的优势, 它使用 GDAL/OGR 库来读取 和写入地理信息数据格式, 相对于 ArcGIS, QGIS 支持的数据格式更多更方便, 大概有

表 1 Leaflet 源码文件组织结构表

\begin{tabular}{|l|l|l|}
\hline \multicolumn{2}{|c|}{ 文件 } & 内容 \\
\hline Core & 核心类 & $\begin{array}{l}\text { 定义面向对象的特性、事件、常用 } \\
\text { 工具函数 }\end{array}$ \\
\hline layer & 地图类 & $\begin{array}{l}\text { 定义地图的属性和行为, 用于初始 } \\
\text { 化和操作地图 }\end{array}$ \\
\hline geo & 地理坐标类 & 定义 WMS、基本图形、图形集合等数据类型 \\
\hline geometry & & 定义像素坐标点、坐标系和投影等 \\
\hline control & 控件类 & 定义控件以及缩放、线、面的工具函数等 \\
\hline dom & DOM 等 4 个常用控件 \\
\hline images & 图片类 & 定义操作 DOM 的工具函数, 用于添素材事件监听的函数等 \\
\hline
\end{tabular}

\subsection{QGIS}

QGIS 是 2002 年开始由世界各地广泛的 GIS 开发爱好者采用 $\mathrm{C}++$ 语言自愿编译完成 的一款开源的桌面地理信息系统软件, 其界 面友好、跨平台性强(支持 Windows、Linnux、 Unix、Mac OSX 等操作系统), 是目前支持 操作系统最为广泛的桌面 GIS 之一。另外, QGIS 还构建了方便用户进行二次开发的 API 库[13]。2017 年为止最新的版本是 QGIS 2.18。

作为开源 GIS, 相对于商业 GIS, QGIS 安装简单快捷、成本低、文件体积小, 占用 内存小, 可在 CPU 被限制的环境下运行。 QGIS[14]可以(1)支持多种矢量图层文件和 栅格数据的读取加载和显示, (2)支持图层文 件的基本操作, 例如: 移动、放大、缩小地 图, 显示当前图层等, (3)支持对地图的编辑 处理、查询与制图等功能, (4)支持插件形式
超过 70 种, 例如 KML、Geojson、txt、dwg 等格式, 使得我们免于纠结于数据格式的兼 容性。

\section{3. 体系框架的设计}

地理信息服务构建与调用采用 $\mathrm{B} / \mathrm{S}$ 端 架构, 分为交互层、服务层和数据层。

交互层的客户端开发平台主要采用利 用 Leaflet 和 JavaScript 技术, 从而实现对地 理信息服务的调用和对功能接口的访问, 使 得用户仅仅通过汶览器就可以对地理信息 进行汶览和操作; 服务器层包含网络服务器 和地图服务器。Web 服务器采用 Apache 来 处理客户端与应用服务器之间的通信, 完成 客户端的请求命令。PHP 和 JavaScript 语言 开发的智联网承担了部分地图服务器的功 能, 对来自于汶览器或者其他应用程序的各 种服务请求进行处理, 并完成对数据库的访 问, 分析处理数据库中获取的相关数据, 并 
将处理的结果以文字、数值、符号、图片等 形式传递给客户端; 数据层则选用 MySQL 数据库作为数据管理平台, 负责数据的管理、 存储任务。

交互层、服务层和数据层三者之间的体 系框架如图 2 所示。

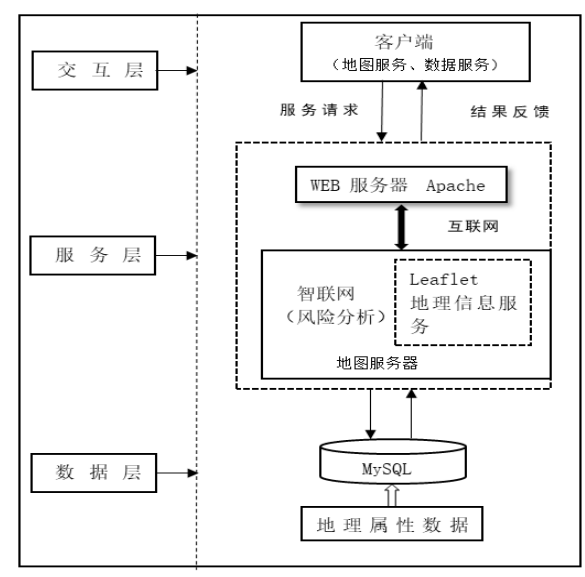

图 2 体系框架图

\section{4. 基于 Leaflet 地理信息服务智联网系统的}

\section{构建}

本文选择四川省三台县永和堰灌区作 为地理信息系统支撑的智联网系统的研究
区域, 根据该区域的自然地理以及自然灾害 风险分析工作的对地理要素的需求, 搭建基 于 Leaflet 的地理信息服务智联网系统。

首先, 采集该地区重点地理要素的矢量 数据, 通过 QGIS 对各个图层进行编辑, 并 导出生成样式文件; 然后通过 Leaflet 对地理 信息进行调用; 最后将基于 Leaflet 的地理 信息服务嵌入智联网系统, 逐个导入各个图 层的样式文件, 同时, 对图层的显示范围、 坐标系等参数进行设置。

\section{1 研究区域自制地图的制作}

根据研究区域的实际状态以及研究目 的和研究需求, 选择并制作相应的图层, 图 层文件的制作在 QGIS 中完成。

本文针对研究目的、研究区域概况、以 及研究需求, 需要制作的图层文件有(1)四川 省三台县行政边界图层 (乡镇级别)、(2)四 川省三台县县域内河流图层、(3)四川省三台 县永和堰灌区重点乡镇图层、(4)四川省三台 县永和堰干渠走向图层、(5)永和堰关键节点 图层。通过 QGIS 的地图制作功能, 以及矢 量化、剪裁、选择、属性编辑、地理坐标系 的配置等功能完成以上矢量图层的制作。 QGIS 中矢量图层添加功能示意图如图 3 所 示。

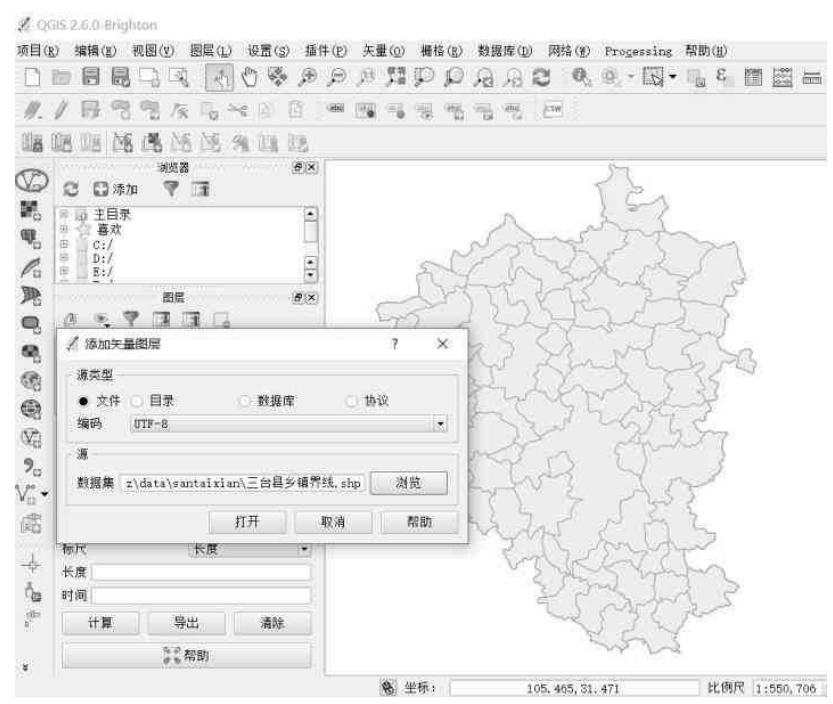

图 3 QGIS 矢量图层添加功能示意图 


\subsection{GEOJISON 文件的生成}

在 QGIS 中选择图层, 通过 QGIS 强大 的格式转换功能, 选择一个图层, 右键选择 “矢量图层另存为”, 将图层另存为 GeoJSon 格式。GeoJSon 生成方式示意图如图 4 所示。 vargeojson; // 申明图层变量

geojson $=$ L.geoJson $(z d,\{$

style: style,

\}).addTo(map); // 需加载的图层名称

(2) 地图缩放控制

Varzoom Control=L.control.zoom(\{position:

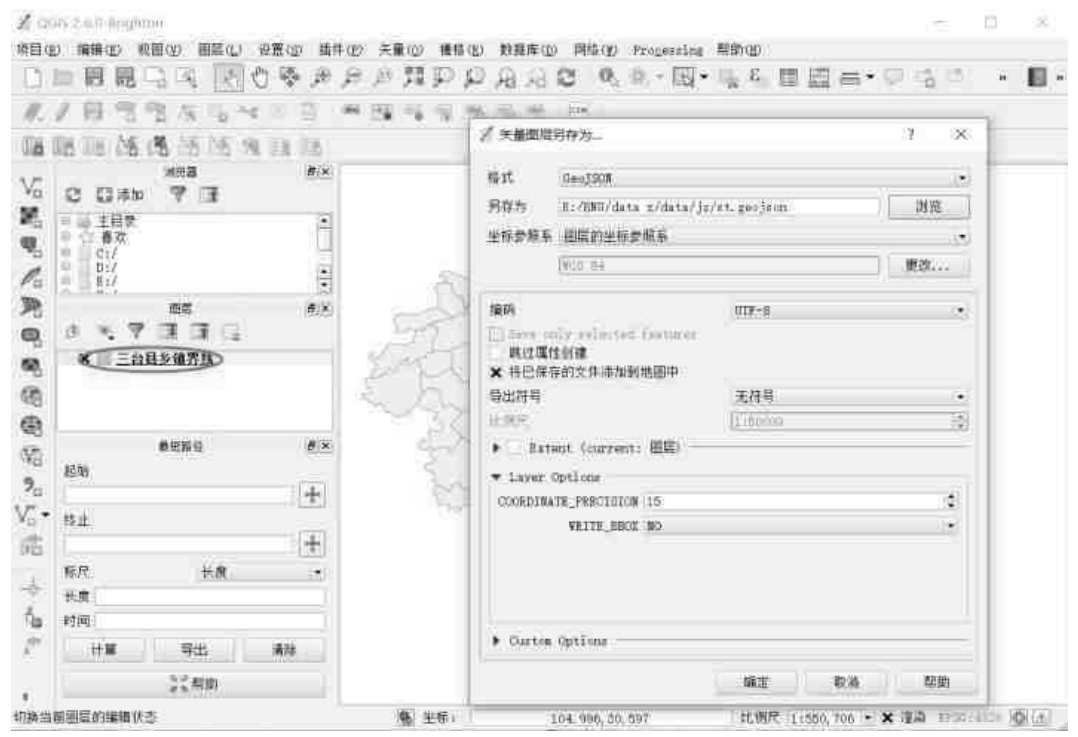

图 4 GeoJSon 生成方式示意图

\section{3 基于 Leaflet 的地理信息服务调用}

本文采用 Leaflet[15]和 JavaScript 技术 对地理信息服务进行调用。Leaflet 的核心框 架是 Map 类, 我们可以形象的将 Map 类 看做一个容器, 然后添加与该地区自然灾害 风险分析需求相应的图层、控件、标记到容 器中。

一般情况下, 在汶览器中显示时, 只有 地图的放大、缩小、移动等基本功能。如果 需要更加复杂的功能, 可根据 Leaflet 提供 的 API, 在此基础上进行拓展, 针对我们的 需求特色来实现所需要的功能。下面简单的 介绍下地图数据源访问、图层控制等基本功 能的接口函数代码。

(1) 网络地图服务的调用

$\operatorname{var}$ map = L.map ('map').set View([31.26,104. 98], 10) ;//设置地图显示的中心点坐标和级 别 'bottom-right'

\}) ;

Map.addControl( zoom Control) ;

(3) 经纬度坐标的获取 functiongetlatlng(e) \{

alert(e.latlng);

\} ;

\section{4 研究区域自制地理信息系统的实现}

\subsection{1 研究区域地理信息服务}

根据自然灾害风险研究需要, 通过 Leaflet实现对研究区域自制地理信息系统的 图层部署和调用, 从而不依赖任何第三方插 件实现研究区域自然地理空间分布状态的 在线服务以及经纬度等位置信息的获取。四 川省三台县永和堰灌区自制地理信息系统 支撑的智联网系统如下图 5 所示。

图 5(a) 展示了研究区域四川省三台县 永和堰灌区的自制地理信息图层, 不用下载 


\section{A}

Advances in Economics, Business and Management Research, volume 66

任何 GIS 软件, 仅需要汶览器就可获取到该 区域的地理信息服务; 图 5(b)展示的该区域 的自制地理信息系统上任何一点的位置坐 标的获取方式, 只需要点击地图上的相应位 置, 该点的经纬度坐标即可出现在经纬度坐 标框内。

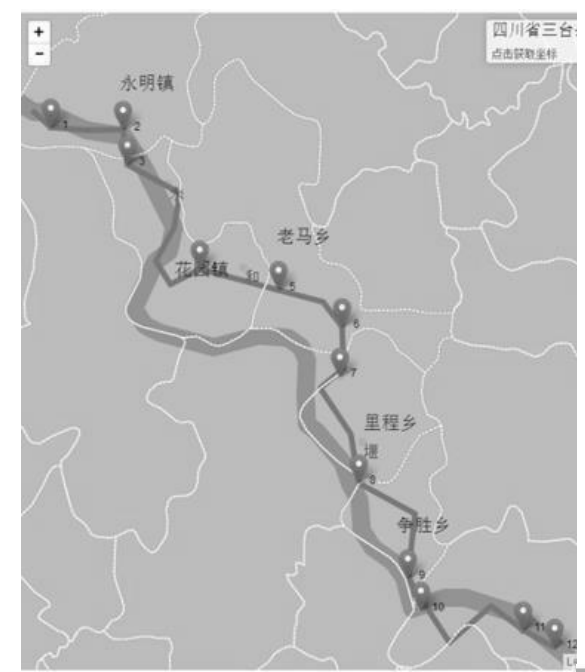

(a) 四川省三台县永和堰灌区自制地理信息图层

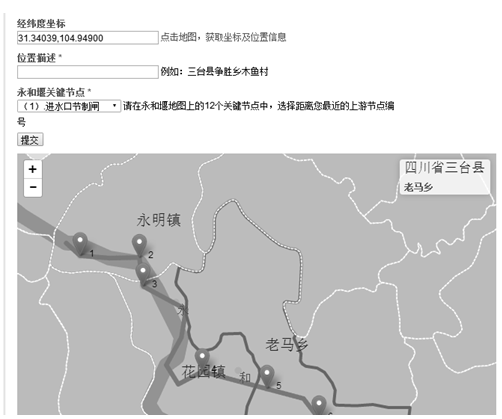

(b) 经纬度坐标的获取展示图

图 5 基于 Leaflet 的四川省三台县永和堰灌区自 制地理信息系统示意图

4.4.2 研究区域脆弱性展示图

通过对重点研究乡镇的自然地理数据、 经济社会数据、灾害损失数据等数据的收集 整理模型计算与处理, 获得研究乡镇的自然 灾害风险分析相关的重要基础数据以及相 对脆弱性数据, 并在结果展示页面中对其展 示和查看。例如在下图 6 乡镇相对脆弱性展
示页面中, 点击花园镇下方的“详情”按钮, 即可获得图 7 中花园镇的灾害风险分析基础 数据以及智联网系统对该乡镇的相对脆弱 性评估值参考, 并且可通过数据下载页面, 通过数据文件描述, 查找可用数据并下载, 为后续的该区域自然灾害风险分析提供基 础数据的支持。

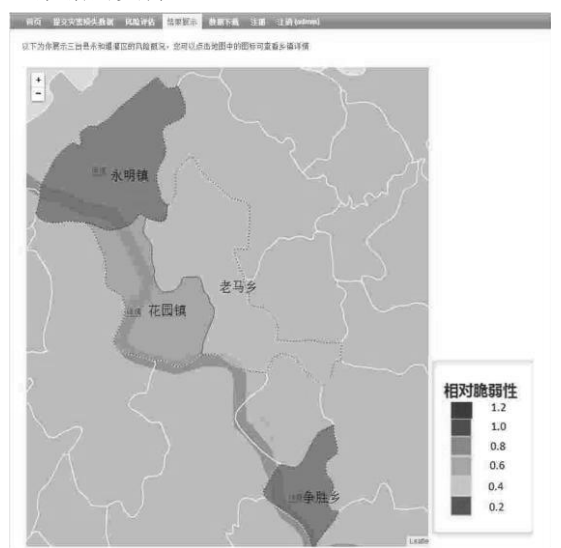

图 6 乡镇相对脆弱性展示图

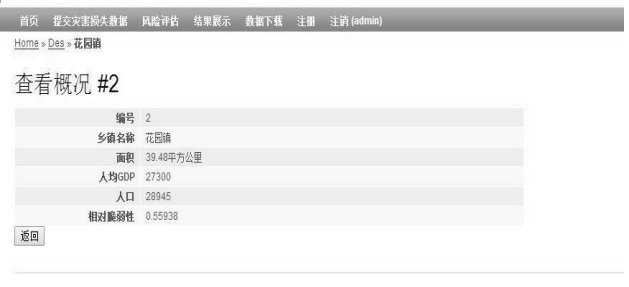

图 7 乡镇基础数据展示图

\section{5.结论}

本文尝试搭建不依赖任何第三方插件, 实现在线地理信息服务的自制地理信息系 统来支撑智联网的在线风险分析工作, 增强 地理信息服务部署的便捷性和对不同平台 的适应性。

通过自制地理信息系统支撑的智联网 在线风险分析系统的体系框架设计, 以及对 系统的开发工具以及系统搭建过程介绍, 通 过实际案例, 为四川省三台县永和堰灌区的 风险分析工作, 专门搭建了自制地理信息系 统支撑的智联网风险分析系统, 实现了研究 区域仅使用汶览器即可实现的地理信息服 务, 证明系统的可行性与操作性。并通过对 
系统中获取灾害损失数据的信息扩散处理, 得到四个重点研究乡镇的相对脆弱性值, 并 以相对脆弱性图的形式展示, 探讨该区域涪 江中上游各个乡镇的灾害风险脆弱性的空 间分布规律，从而对县域内不同乡镇的灾害 风险差异性进行解释, 为灾害风险分析工作 的精准化开展提供支持。

\section{参考文献}

[1] 刘岳峰. 地理信息服务概述. 地理信息 世界, 2004,2(6):26-29.

[2] 葛文. 地理信息服务发现方法研究. 郑 州:解放军信息工程大学, 2012 .

[3] 周成虎. 我国地理信息系统技术发展的 机遇与挑战. 科学时报, 2007.

[4] 徐永龙. 基于 Flex 和 ArcGIS Server 的 WebGIS 实现研究. 西安: 长安大学, 2011.

[5] 李光师. 基于开源平台构建 Web GIS 应用系统. 测绘科学, 2011,36(6):259261.

[6] 赵芳. 基于开源 WebGIS 平台的地理信 息系统应用研究.郑州大学, 2010 .

[7] 刘光,曾敬文. Web GIS 原理与应用开 发.清华大型出版社, 2016:16-19.

[8] Huang C F. Internet of intelligences in risk analysis for online services. Journal of Risk Analysis and Crisis Response, 2001,2(1); 110-117.

[9] Huang C F. Internet of intelligences can be a platform for risk analysis and management. Human and Ecological Risk Assessment, 2015,21(5):1395-1409.

[10] 黄崇福. 用智联网缓解风险分析中的 4 个问题. 自然灾害学报, 2014(02):1-7.

[11] 黄崇福. 自然灾害风险评价理论与实践. 科学出版社发行处出版社, 2006.

[12] An open-source Java Script library for mobile-frierdlyin-teractivemap[EB/OL]. http://Leafletjs.com.

[13] 陈卫林,郑礼全. 基于开源 QGIS 源码编 译的二次开发方法的探究. 北京测绘, 2015(06):71-74.
[14] 胡达天,胡庆武. 基于开源系统的跨平 台地图客户端开发. 测绘科学, 2015, 40 (7): 142-145.

[15] Zuo M Y. FrameWork for Integration of SOA and Web 2.0. Journal of Southeast University (English Edition), 2003, 23(3): 381-384. 